\title{
Evolving a Real-time Evacuation for Urban Disaster Management
}

\author{
Keith J. Drew \\ University of Idaho \\ 895 Perimeter Drive \\ $\begin{array}{cc}\text { Moscow, Idaho } 83844 & \text { Moscow, Idaho } 83844 \\ \text { keithd@vandals.uidaho.edu heckendo@uidaho.edu }\end{array}$
}

\author{
Ahmed Abdel-Rahim \\ University of Idaho \\ 895 Perimeter Drive \\ Moscow, Idaho 83844 \\ ahmed@uidaho.edu
}

\author{
Homaja Pydi Kumar Marisetty \\ University of Idaho \\ 895 Perimeter Drive \\ Moscow, Idaho 83844 \\ mari7627@vandals.uidaho.edu
}

\author{
Anton Stalick \\ University of Idaho \\ 895 Perimeter Drive \\ Moscow, Idaho 83844 \\ stal6565@vandals.uidaho.edu
}

\begin{abstract}
In an urban disaster it is important to efficiently evacuate people to safety. We use evolution strategies and a probability model to route the population by optimizing their safety. The algorithm is designed to use the strengths of evolutionary computing to repeatedly optimize an evacuation under the dynamics of a disaster such as accidents blocking critical roadways, bridge collapses, debris closures, changes in safety, and people not following evacuation directions. Our model is unconcerned with specific evacuation routes but rather evolves a robust cloud of probabilities to represent best directions of escape. We show that maintaining a population of diverse solutions may allow for rapid adaptation as a disaster unfolds. The core optimization algorithm is tested using challenging test cases as well as real-world data.
\end{abstract}

\section{Keywords}

Evacuation Planning; Evolution Strategies; Traffic Congestion, Safety Optimization, Traffic Assignment

\section{INTRODUCTION}

Natural and man-made disasters can rapidly render portions of large urban areas unsafe for people. To save lives, emergency managers may order time sensitive evacuation from unsafe areas. This can lead to overloading of transportation networks resulting in the failure to evacuate all of the population from the unsafe areas and large loss of life. Even if an evacuation is planned for long before it is needed, numerous unforeseen events may occur during the evacuation such as accidents blocking critical roadways, bridge collapses, debris closures, and flooding can cause evacuation failure and loss of life. Changes in safe zones can occur as

Permission to make digital or hard copies of all or part of this work for personal or classroom use is granted without fee provided that copies are not made or distributed for profit or commercial advantage and that copies bear this notice and the full citation on the first page. Copyrights for components of this work owned by others than ACM must be honored. Abstracting with credit is permitted. To copy otherwise, or republish, to post on servers or to redistribute to lists, requires prior specific permission and/or a fee. Request permissions from permissions@ acm.org.

GECCO '17, July 15-19, 2017, Berlin, Germany

(c) 2017 ACM. ISBN 978-1-4503-4920-8/17/07 . \$ $\$ 15.00$

DOI: http://dx.doi.org/10.1145/3071178.3071254 well such as failure to predict the level of storm surge in a hurricane or wind changes in a poison gas leak. Finally, the human factor of people simply not following evacuation instructions may also lead to the need to repeatedly re-optimize to avoid congestion resulting in loss of life.

Unlike some previous work, we see the evacuation problem not as getting individuals to their predefined safe destinations, nor as distributing the people across a small set of safe destinations but rather as getting people to regions of safety indicated by the level of safety of the nodes in the transportation network. For example, the problem may be to get people to the half of the city that is higher ground or safety may even be equated with elevation of each intersection above sea level. Often city emergency planners have safety maps for various scenarios. There is also a capacity constraint on nodes so that solution must conform to physical capacities of nodes and edges. We use both real and test city networks. Traffic congestion is modeled using classical traffic formulas. This problem specification is very general in that we optimize routing based on a arbitrary safety function which can be established for each type of event and so is not necessarily limited to disasters, although this paper focuses on evacuation planning and response.

We approach the problem of evacuation as a dynamic problem that needs to be repeatedly solved as a disaster unfolds to handle the unforeseen changes in the problem parameters. This we feel is an added strength of an evolutionary solution. A diverse population in nature or in silico maintains a set of contingent genes that help it adapt to changing environments. We will attempt to exploit this.

Our solution is to treat the evacuation as a problem of optimizing the sum of the safety of all the people at a time $t$. While more complex and interesting optimizations can be done, we believe that this building block optimization is the "proof of concept" we will base future work upon. We use an evolutionary approach in which we maintain a population of potential solutions and evolve an evacuation plan (see details below). We hypothesize first: that with an effective evacuation representation, we can efficiently solve a wide variety of evacuation scenarios using an Evolution Algorithm. Second, if during the evacuation unexpected changes in the transportation network, the safety of regions, or the evacuee's position occurs, the population of evacuation plans in the 
algorithm can be used to more quickly compute a response to the change than if the response was evolved from a restart of the optimization. That is, as one would expect, the population acts as a reservoir of innovation for the adapting of an evacuation to changing conditions. Experiments are performed on a variety of problems to test and support both hypotheses.

We proceed as follows: Section 2 discusses previous work, Section 3 discusses our algorithm, Section 4 describes in detail a suite of different problems to challenge our algorithm, and Section 5 presents a summary of conclusions.

\section{BACKGROUND}

Evacuation planning is not a new topic. Previous work has addressed problems varying from room or building evacuation [1], [2], to city or region evacuation [3], [4], [5]. Optimizing traffic while considering the dynamics of traffic interaction (e.g. congestion [6]) is known as Dynamic Traffic Assignment (DTA) [7]. Our approach models evacuation as a DTA problem with the single objective of optimizing the safety of the evacuees. Some authors consider evacuation as a multi-objective optimization problem [8], [9].

Saadatseresht et al. optimize evacuation with respect to capacity and nearness of safety zones, while Yuan and Han suggest that evacuation is an inherently multi-objective optimization problem, and recommend optimizing space-based risk and travel time.

Stepanov and Smith also approach evacuation planning as a multi-objective optimization problem in [10], where they use Integer Programming instead of evolution. A routebased approach is used. This has the limitation of having to establish routes for each individual, creating a specific solution that may be ignored.

Attempts have been made at handling roadway congestion using genetic algorithms, as well. In Dezani et al. [11], Petri net analysis is used to evaluate fitness in a GA designed to optimize routes for urban traffic, strictly to reduce roadway congestion in real-time. Their approach is not multiobjective, and optimizes routes for shortest time. However, their approach also focuses on route-based solutions for individuals, and optimizes for minimization of travel time alone.

Our solution focuses on safety optimization, without determining routes for each traveler. This provides a generalization for DTA, removing the requirement that each vehicle follow a specific path.

\section{APPROACH}

We use an Evolution Strategy (ES) [12] to evolve sets of probabilities that determine the probability $p_{n e}$ of traffic at node $n$ exiting on edge $e$ with the constraint that

$$
\sum_{e \in E(n)} p_{n e} \leq 1 \forall n
$$

where $E(n)$ is the set of edges leaving node $n$. The amount of probability left between the sum above and 1 is the probability of remaining at that node before trying again to leave the node. The fixed cycle-time is specified for each node when the node is defined. This approach does not build explicit routes but rather relies on probabilistic routing at each intersection to move traffic to safety. An advantage of this is that local information about the best route to safety is stored at each intersection. The disadvantage is that in this first implementation, the probabilities are static and only change with re-optimization.

The genome for the ES is the vector of the probabilities given by the list of $p_{n e} \forall n$. Fitness at time $t$ is computed:

$$
\text { fitness }(E, t)=\frac{1}{\|E\|} \sum_{a \in E} S(L(a, t))
$$

where $E$ is the set of all evacuees, $t$ is time, $S(\ell)$ is the safety of location $\ell$, and $L(a, t)$ is the location of agent $a$ at time $t$ which can be a node or edge. Because this is an average of safeties, the fitness may make trade-offs based on level of safety across the whole population. This is a practical consideration for the user of the application.

We chose to use an $E S(\mu+\lambda)$ algorithm. For many of our tests we used, $\mu=100, \lambda=100$, and $\sigma=0.1$ (see specific test results). Using the + operator, the child population competes against the parent population for survival. This is performed using a simple sort operation, followed by removal of individuals with the lowest $\lambda$ fitness scores. Mutation probability $P_{\text {mutation }}$ for a selected genome is 0.5 for each real number in the genome. Mutation happens by mutating the value by a normal distribution with $\sigma$. Unlike many ES there is also crossover with probability $P_{\text {crossover }}=0.2$. Crossover is uniform crossover respecting that all probabilities for a given node change together.

It is important to note that time appears in two contexts in this paper. First, simulation time refers to the amount of time simulated during a fitness evaluation (the $t$ in 2). Second, the amount of real-world time it takes to run the model for a given problem, referred to as wall-clock time.

\subsection{The SLang Language}

We developed a language called SLang (Simulation Language) that can be used to specify cities and optimizations so that tests can be easily generated, run, saved and shared. SLang is used to specify such things as city topology, the level of safety of intersections, and agent distribution. SLang also specifies experiment parameters for the evolution strategy algorithm and simulation of traffic. SLang is also used to control I/O features of the code.

SLang commands are used to specify all necessary information needed by a model. Therefore, in our model we use SLang to indicate ES parameters such as population size, $\sigma$ and the maximum number of generations. SLang is also used to specify the traffic network data, indicating freeflow time, simulation time, and more.

\subsection{Simulation}

During simulation agents move through the provided topology, according to the current set of probabilities being evaluated. Agents are stored in a priority queue, sorted by time of next arrival at an intersection. Time progresses in steps governed solely by the next arrival at a intersection.

The simulation operates by dequeuing an agent from the queue, checking the arrival time of that agent compared to the current time in the simulation, selecting the next edge that agent will take based on the probabilities for the node the agent is currently at, calculating the traversal time of the selected edge, then re-queuing the agent. This cycle repeats until the next agent in the priority queue has an arrival time exceeding the total simulation time (see Figure 1).

We defined the travel time $t$ between nodes using the clas- 


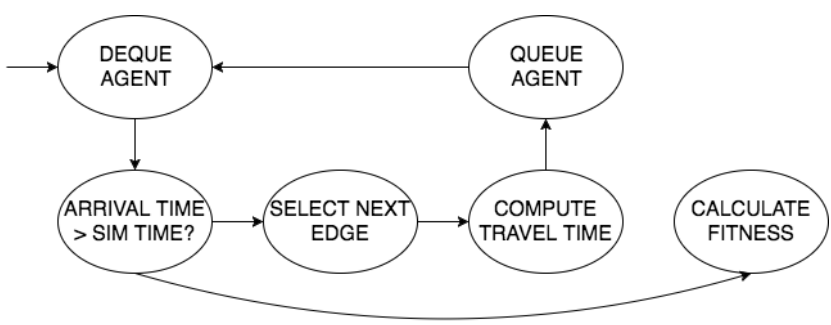

Figure 1: A diagram of the how time progresses using a priority queue, during simulation.

sic Bureau of Public Roads (BPR) formula [13]:

$$
t=f+b\left(\frac{c}{c_{\max }}\right)^{\beta}
$$

where $f$ is freeflow time of the edge, which is the time required to travel the edge in ideal conditions, typically meaning no other traffic. For our simulations all time is measured in hours. $c$ is the current number of evacuees on the edge, while $c_{\max }$ is the maximum number of evacuees that the edge can handle per unit time (hour). This is a combination of speed limit, number of lanes, road condition, and length of road. $b$ and $\beta$ are tuning variables used to fit real data measured from actual roadways. For our purposes we used the default values of $b=4.00, \beta=0.15$, and $c_{\max }=1000$ but is dependent on road size, speed limit and length. Note that the formula does not include explicit distance or speed limit values, as those values are implied by the freeflow value, $f$, as a result of $t=$ distance/speed.

Historically, in normal traffic conditions with working traffic lights, $c_{\max }$ must account for total throughput per unit time. For instance, the value is reduced from 1000 by a proportion equivalent to the proportion of "green time" of the traffic light at the intersection.

Once the simulation is complete, the set of probabilities used in the simulation are evaluated for their fitness. Fitness is a sum of the safety of all the agents (see Equation 2). The maximum safety for any location is 1.0. Because fitness is currently evaluated only at the end of the simulation, fitness is a measure of safety at a specified time $t$.

This fitness function was chosen to allow adaptability to any disaster since safety can be designated by any factor. For example, a flood or tsunami event might measure safety as both distance from the event, as well as elevation. An earthquake may quantify safety as distance from tall structures or other hazards. Some events will use distance alone, but other events may have more complex safety functions.

In summary, a fitness evaluation is computed as follows:

\section{Initialization}

The set of probabilities to be evaluated is loaded into the city topology; the city has been defined in SLang prior to any fitness evaluations and the set of probabilities must match that topology.

Agents are initialized in their starting locations and times; their initialization values are specified in SLang before any fitness evaluations are made. Typically, agents are initialized with an arrival time of zero seconds. Finally, the current time is set to zero seconds. This value is replaced with each next agent evaluated, and is replaced with the next agent's arrival time.

\section{Simulation}

The main activity during simulation consists of popping agents from the priority queue one at a time, evaluating their next move and arrival time at the next node, and replacing them in the priority queue at the appropriate location. In the case where an agent chooses to stay in a node, the agent's next time of arrival is specified by the node's wait time field, and is generally fixed.

\section{Fitness}

The fitness of the set of probabilities is calculated when the simulation time has expired, using Equation 2.

\section{EXPERIMENTS}

A series of experiments were performed to test our software and hypotheses. The tests included validation tests to show that the code works in simple transparent problems, dynamic tests to show that the code can use populations to adapt to changing problem parameters such as capacity, comparison tests to compare our work to the work of others, and scaling tests to see if the code will work for topologies and traffic found in a real city. While we ran many tests, due to publication size, only a subset of tests is given in this paper.

\subsection{Validation Tests}

To validate our model we performed a variety of simple transparent tests. First, we created a test with a $5 \times 5$ grid, with all edge and node variables equal. Agents were initialized to begin in the top left corner of the grid, while safety was only in the two nodes in the bottom right of the grid (see Figure 2). The program quickly routed all evacuees to safety.

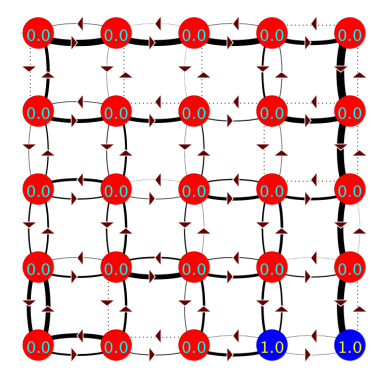

Figure 2: Simple $5 \times 5$ grid validation test. The heavier weight edges in the figure show higher probabilities. The thinnest lines are generally very near zero but are just drawn to show the topology. The numbers in the nodes are level of safety. Unless otherwise stated, best case of several is shown.

To validate our model we tested that capacity and freeflow constraints effected our results as expected. We recorded the path to safety indicated by highest probabilities calling it the selected path. We then repeat the experiment by creating a 
single path from danger to safety by reducing the freeflow time of the edges along the selected path, while increasing freeflow along every other edge in the graph the path is easily found, as it should be. Similarly, if we increase maximum capacity along the selected path, while decreasing maximum capacity along all other edges, the path is again easily found.

We then constructed a maze as seen in Figure 3. The path indicated in the figure was marked in one test by high capacity and marked by low freeflow time in the other test. Notably the safety was zero except for the safe zone at the end. Both tests found the easier routes highlighted in the figure. As the number of evacuees increases past the limit of the easy path side streets become used to share the load.

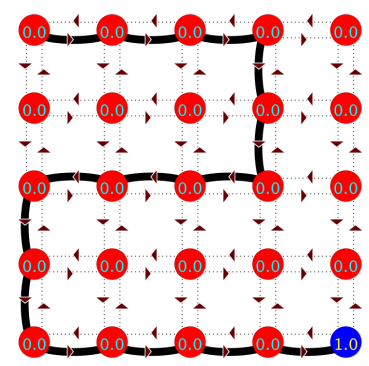

Figure 3: A cartoon of $5 \times 5$ maze test showing the route of least resistance. In this case, these are not a probabilities but rather just an indication of where we tried to make the roads faster or higher capacity.

\subsection{Dynamic Tests}

The following experiments test if our model accommodates dynamic events during an evacuation. We hypothesize that if probability distributions are optimized in advance, they can be used to initialize an evolution strategy (ES) to reduce optimization time, both in real time, and number of generations without loss of accuracy. The results of these tests demonstrate that pre-optimization leads to faster adaptability in response to real-time events. This is a very important feature for a real-world evacuation management system.

For these experiments we make several assumptions. First, we assume changes are not significantly large. We assume an algorithm's population will have genetic contingency information usable for similar problems. For example, we assume that from one optimization to the next during an evacuation, we won't suddenly switch from West side of the city is safe to East side is safe. In fact, in such a case where the graph characteristics have been changed significantly, re-optimization using a previous solution may take significantly longer than using random initialization. The events we are concerned with are changes in topology, safety zones, capacity, and agent distribution.

To test our hypothesis, we perform two initial optimizations (case 1) we optimize a population of solutions that are initialized with random values for the initial graph configuration and (case 2) we optimize a population of solutions that are initialized with random values for the graph config- uration after some change in topology, safety, capacity, or vehicle distribution has been modified. Finally, (case 3) we use the final population from the first optimization to initialize the starting population used to optimize the second graph configuration. Results will be labeled 1, 2, or 3 corresponding to the three different cases. For each test, the ES parameters were kept the same for the sake of comparison. The parameters are $E S(100+100), P_{\text {crossover }}=0.2, P_{\text {mutation }}=$ $0.5, \sigma=0.1$

\subsubsection{Adapting to Capacity Changes}

During an evacuation event a roadway may see a change in capacity as a result of becoming partially blocked by debris, accidents in one or more lanes on a multi-lane road, or as a result of other unpredictable events. In such a case our model must be able to quickly adapt through re-optimization. We predict that using pre-optimized traffic probability distributions will allow for quick adaptation. To test this prediction we run three tests. First, we create a topology with a maximum capacity of 1000 agents per unit time for all edges, $G_{1}$. Next, we create a topology that is identical, except that maximum capacity is lowered by 100 for each edge, $G_{2}$.

We then optimize both of these graphs using our models and record the average number of generations until maximum fitness is achieved. We also store the population of solutions found from $G_{1}$, as they will be the starting population for optimization in the next test. For the third part of the test we re-optimize $G_{2}$, starting with the solutions from $G_{1}$. We record and compare the number of generations needed to reach maximum fitness below. The probabilities are graphically represented in Figures 4 and 5. Since the landscape is flat and load is light, many evacuations plans are equivalent and so variation in the probabilities occur. Solution time is shorter in case 3. Results are shown Table 1.

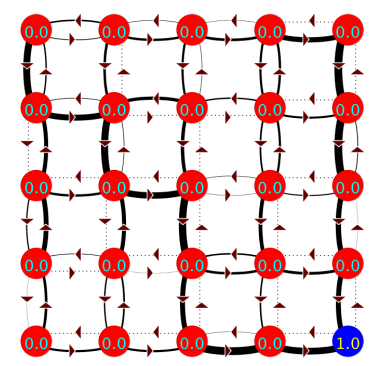

Figure 4: $5 \times 5$ capacity test results for case 1 .

\subsubsection{Adapting to Topology Changes}

Another event that might occur during an evacuation is a change in topology, meaning some route or partial route becomes unusable, such as a bridge collapse or a road becoming flooded or completely blocked by debris. It is important that our model handles such changes quickly. We make a similar prediction to the capacity change test as well. Note that a change in topology is a more extreme version of a change in capacity where maximum capacity is reduced to 


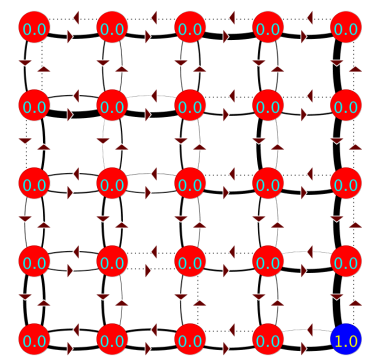

Figure 5: $5 \times 5$ capacity test results for case 2 .

zero for a roadway. We again run three tests but using the bridge topology. The first test is on $G_{1}$, which represents three bridges connecting two areas, with safety being on the side opposite where vehicles are started for these tests (See Figure 6). $G_{2}$ for this test is identical to $G_{1}$, except with an edge on the center "bridge" removed representing a collapse. The first two graphs are optimized from random traffic assignment probabilities. We then use the solutions from the optimization of $G_{1}$ to optimize $G_{2}$, and record the number of generations for comparison (See 7). Results are shown Table 1. Solution quality is similar but time to solve is substantially shortened.

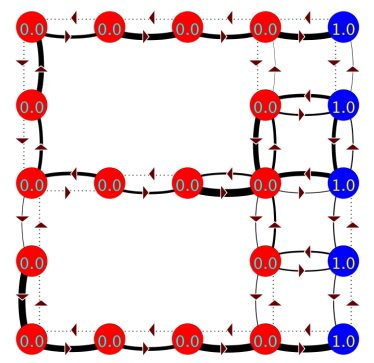

Figure 6: The three bridge test. Probabilities for bridge intact and random initialization.

\subsubsection{Adapting to Agent Distribution Changes}

In an evacuation, it is expected that individual drivers will ignore evacuation instructions and instead upon arriving at and intersection rely on their experience driving in the area. This is expected because people are under stress and optimal traffic assignments may produce non-intuitive results in an attempt to balance traffic load on many streets at once. In such cases, we must adapt and adjust based on where vehicles actually are not where we expect them to be. This is another reason why it is crucial to continually re-optimize to adapt to the unforeseen. We make a similar prediction here - our model will find an optimal solution faster if initialized with a pre-computed solution for a similar traffic distribution. Again, we use three tests. All tests

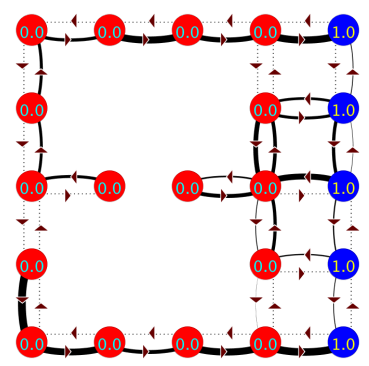

Figure 7: The three bridge test. Probabilities for bridge destroyed and population initialized with population from previous solution.

use an identical topology, but $G_{1}$ has traffic distributed differently than $G_{2}$. The first two runs optimize for different traffic distributions, starting with randomized traffic assignment probabilities. The third run uses the optimized traffic assignment probabilities from the first test, but with the traffic distribution of the second run. The traffic probability distributions are qualitatively not different but the time to solve is much lower. Results are shown in Table 1.

\subsubsection{Adapting to Safety Changes}

During many evacuation events, the threat creating the need for evacuation is not static. Examples include hurricanes, floods, tsunamis, poison gas. As a result of the dynamic nature of the threat, it is imperative that our model handles changes in safe areas quickly. We predict that our model handles changes in safety quickly, again provided the change is not too great. Three runs are used to perform this experiment. The first and second are again identical in topology (see Figure 8). In the case 3 test the safe nodes move over one node (see Figure 9). The optimized traffic assignment probabilities from run one are used to initialize the third run, which has safety in the same location as run two. Results are shown Table 1 . Safety was easily solve quickly. Perhaps a more difficult safety test will tease out the difference between case 1 and 3 for this problem.

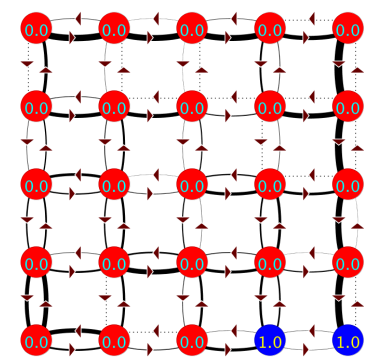

Figure 8: $5 \times 5$ safety test for case 1 . 


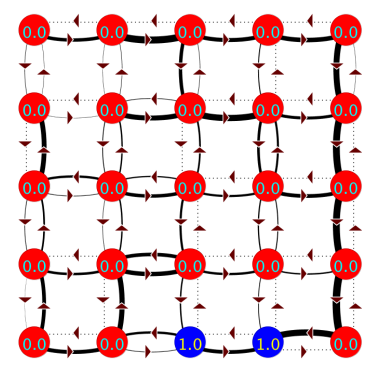

Figure 9: $5 \times 5$ safety test for case 3 .

Table 1: Generations to Complete Optimizations in Dynamic Tests: Capacity, Topology, Agents, Safety. In all cases the suffix numbers 1, 2, or 3 represent the three cases in each of the dynamic tests. 100 runs of each case were performed. "Gens" is generations and $t_{\text {hours }}$ is the simulated time.

\begin{tabular}{l|cc|cc|c}
\hline \hline Test & Avg. Gens & $\sigma_{\text {Gens }}$ & Avg. Fit & $\sigma_{\text {fit }}$ & $t_{\text {hours }}$ \\
\hline Capacity1 & 47.75 & 5.208 & 1.0 & 0.0 & 1.0 \\
Capacity2 & 55.1 & 6.64 & 1.0 & 0.0 & 1.0 \\
Capacity3 & 4.35 & 2.19 & 1.0 & 0.0 & 1.0 \\
\hline Topology1 & 73.54 & 15.835 & 1.0 & 0.001 & 0.5 \\
Topology2 & 69.92 & 13.9 & 0.998 & 0.009 & 0.5 \\
Topology3 & 7.01 & 4.574 & 1.0 & 0.0 & 0.5 \\
\hline Agents1 & 77.99 & 9.605 & 0.999 & 0.003 & 0.7 \\
Agents2 & 89.69 & 8.577 & 0.998 & 0.004 & 0.7 \\
Agents3 & 30.5 & 8.325 & 1.0 & 0.0 & 0.7 \\
\hline Safety1 & 43.02 & 8.359 & 1.0 & 0 & 1.0 \\
Safety2 & 40.855 & 8.2 & 1.0 & 0 & 1.0 \\
Safety3 & 34.5 & 4.574 & 1.0 & 0 & 1.0 \\
\hline
\end{tabular}

\subsubsection{Summary}

In the dynamic tests we adjusted one of four attributes to represent events that can change a transportation network in an evacuation. Graphs shown in Figure 11 show that priming the population with a population that produced a successful solution for the pre-event problem produces a solution more quickly than just starting the optimization over. Figure 10 shows the quality of the answers is substantially the same. We believe that as long as the change is modest the population contains "contingency genes" that will help speed the adaption to the new problem. These results are encouraging, but we are following with more focused tests in our future work.

\subsection{Comparison Test}

Here we compare our model to the approach used by Bazzan et al. in [3]. In their paper they seek to minimize average travel time by evolving traffic assignments through the network, given a set of vehicles, origin/destination pairs for those vehicles, and a set of $k$ shortest paths. They use a classic graph from [14], (see Figure 12) with the freeflow times specified. The way we model capacity is different, but com-

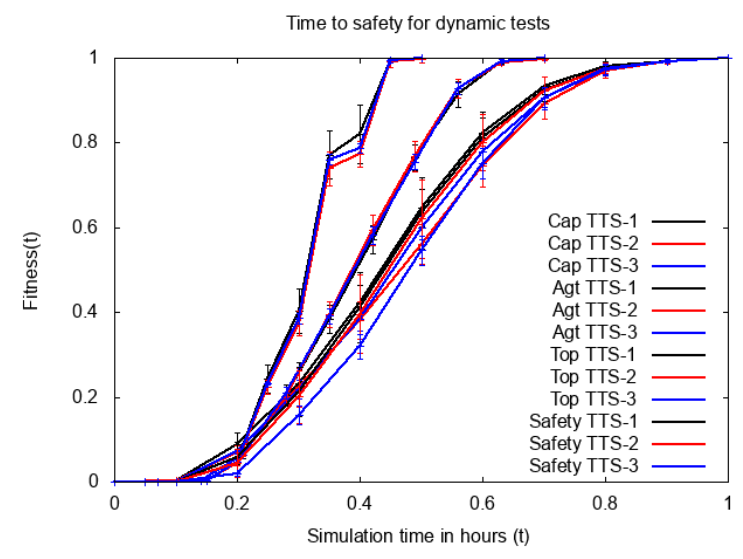

Figure 10: A comparison of the quality of answers for the four dynamic tests. This indicates the answers are of comparable quality.

parable. We seek to compare our results to provide some context of how our model holds up compared to other work.

In their experiment they run $k$ origin/destination pairs, with $k$ ranging from 2 to 16 . We compare to the case where $k=4$. We run our experiment with two vehicle origin nodes, corresponding to their origins, with two safe nodes, corresponding to their destinations. We let our model optimize for safety, and provide the results and comparison below. Their evacuation (network clearance) time is significantly lower. We believe this is probably due, in part, to differences vehicle scheduling and is an area for future analysis. While our model does implicitly find routes to safety, the routes found are not as fast as those found by Bazzan et al. The average time to safety (TTS), with standard deviation is shown in Figure 13.

\subsection{Scaling Tests}

To test the ability of our model to handle real-world urban evacuation problems, we used traffic model data for Boise, Idaho, a city with approximately 650,000 people. Our goal was to discover the wall-clock time it takes to optimize such a city, using our model. For this experiment we made simple assumptions about graph properties where we didn't have accurate data (freeflow time, maximum capacity, etc). Our data includes 283 nodes with 457 edges. These do not represent every road and intersection in Boise, but rather many of the artery roads and feeders (see Figure 14). We believe the vast majority of the evacuee's time will be spent on these roads and so the test is a realistic estimation of evacuation time. We feel that Boise is a good representation of a real example, as it includes a mix of urban downtown areas and suburban neighborhoods.

We ran a set of 6 experiments 10 times each, and averaged the wall-clock time each experiment took. Each experiment used the same ES and city parameters, varying only in the way and number vehicles were distributed. Each experiment was initialized with a base-set of agents (representing 10 vehicles each) at each node. This base-set is used to ensure agents begin at every node, thereby applying evolutionary pressure across the whole genome. Not applying pressure to probabilities at each node could produce dangerous re- 


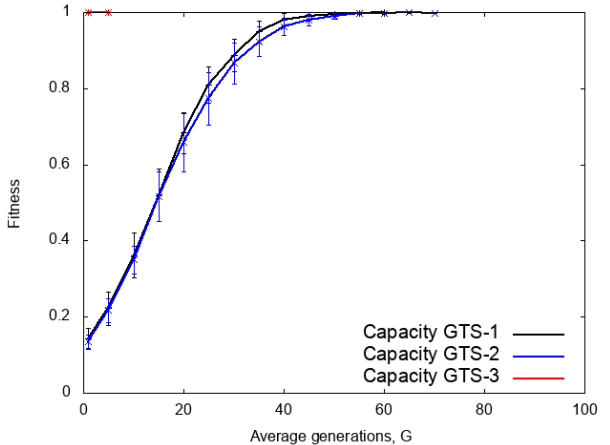

(a) Capacity

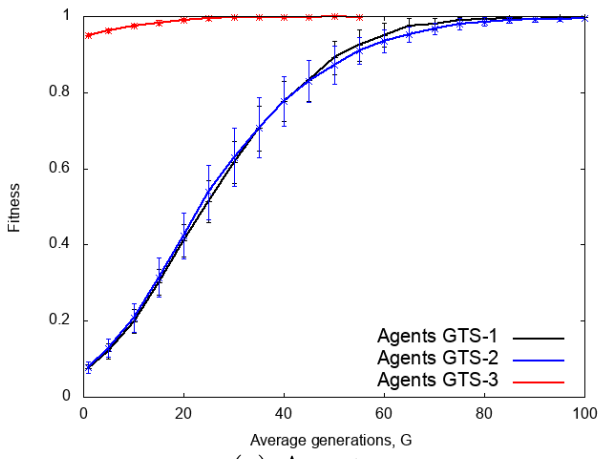

(c) Agents

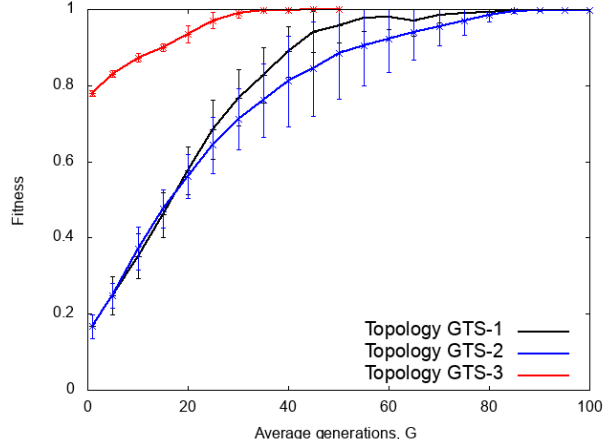

(b) Topology

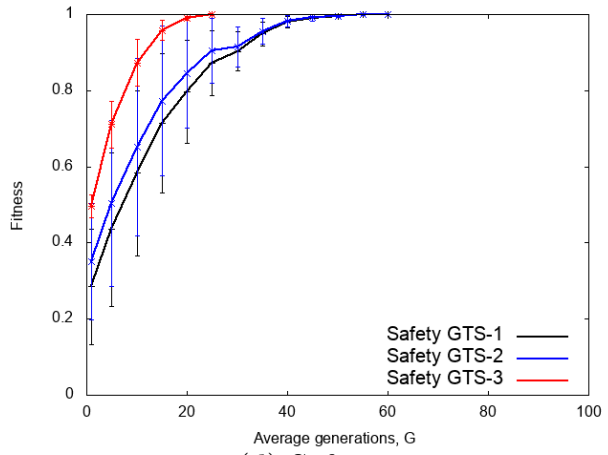

(d) Safety

Figure 11: The speed of solution for the four dynamic tests and across cases 1, 2, and 3. Generations along $\mathrm{X}$-axis and fitness along $\mathrm{Y}$-axes. Values averaged over 100 runs.

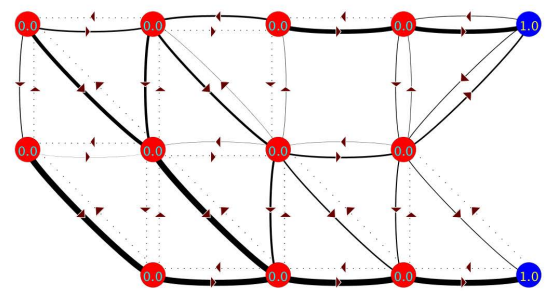

Figure 12: The network from Bazzan et al. 2014.

sults in a real-world application, as nodes may end up with traffic assignments that are no better than random. Vehicle group starting locations are random in each test. Vehicles are grouped to improve run time. Each experiment simulated one hour of traffic. The number vehicles, number of groups, size of each group, and wall-clock run time are shown for each experiment in Table 2. The experiment shows that for a real world city of over a half-million people execution times on the order of minutes is a reasonable expectation.

\section{CONCLUSIONS}

In conclusion, we have evolved static probability distri-

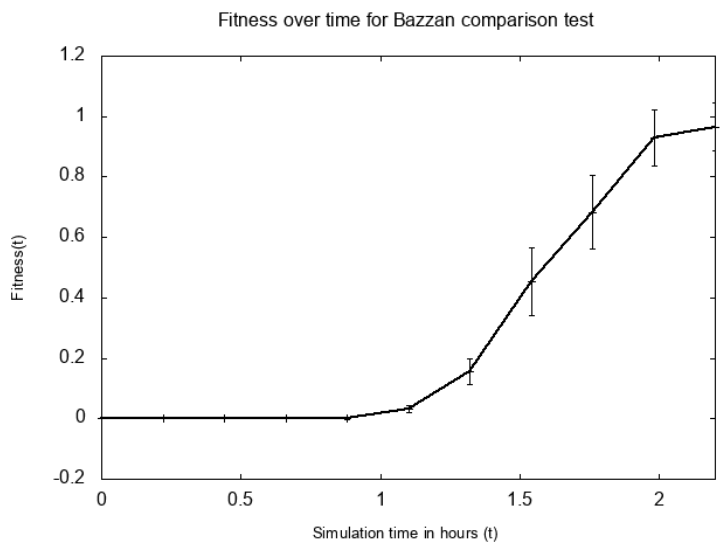

Figure 13: Performance of our algorithm on the Bazzan test averaged over 100 trials.

butions that are shown to provide routing to safe areas in reasonable time. Further, the solutions used are provided as initializations for evacuation parameter changes, which reduce the optimization times of the updated scenarios. This makes an evolution based real-time continuous optimization of an evacuation feasible. The main advantages of our proposed model includes a robust method for adapting to the dynamic conditions of a real disaster, relatively fast computation time, and robustness achieved through both evolution 


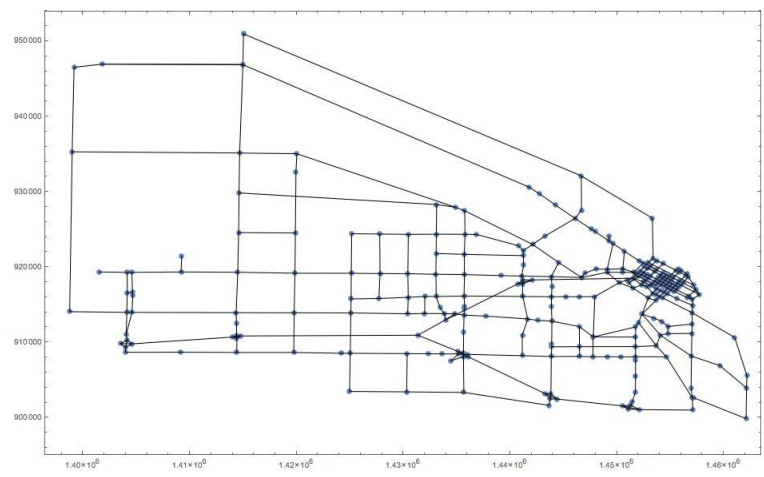

Figure 14: The Boise Transportation Network. Notice the dense packed streets in the downtown area. The map is of an area approximately ten miles wide.

Table 2: Time to solve the Boise problem. Vehicles is the number of vehicles being evacuated. Num Groups is the number of randomly placed groups of agents in the city above the groups that are evenly distributed across the 283 nodes of the city. Group size is the number of individuals in each group or agent. It defines the grouping of the agents. For timing the runs were performed using Centos Linux system running on $2.4 \mathrm{GHz}$ Intel Xeon X56xx processors.

\begin{tabular}{lccc}
\hline \hline Vehicles & Num Groups & Group Size & Run Time (min) \\
\hline 2,830 & 0 & 10 & 3.5 \\
2,930 & 10 & 10 & 3.28 \\
3,830 & 10 & 100 & 3.5 \\
12,830 & 100 & 100 & 4.4 \\
102,830 & 100 & 1000 & 4.25 \\
$1,002,830$ & 1000 & 1000 & 19.05 \\
\hline
\end{tabular}

and a vehicle distribution model, instead of a more ridged point-to-point routing for individual agents.

Future work includes the use of OpenStreetMap [15] for generating graphs representing urban areas. Also, self-adaptive parameters for the ES algorithm, diversity promotion, multiobjective optimization, and novel mutation operators.

\section{ACKNOWLEDGMENTS}

This material is based in part upon work supported by the National Science Foundation under Cooperative Agreement No. DBI-0939454. We would like to thank the U.S. National Science Foundation (NSF), the State of Idaho, and the M.J. Murdock Charitable Trust, for making this research work possible. NSF under CyberCorps ${ }^{\circledR}$ awards 1027409 and 1565572 and BigSTEM award 1229766. Idaho under IGEM grants. Any opinions, findings, conclusions, or recommendations expressed in this material are those of the author(s) and do not necessarily reflect the views of the National Science Foundation or the State of Idaho. We thank Michigan State faculty member Dr. Kalyanmoy Deb for his collaboration in this research.

\section{REFERENCES}

[1] P. A. Thompson and E. W. Marchant, "A computer model for the evacuation of large building populations," Fire safety journal, vol. 24, no. 2, pp. 131-148, 1995.

[2] S. Gwynne, E. Galea, M. Owen, P. J. Lawrence, L. Filippidis et al., "A review of the methodologies used in evacuation modelling," Fire and Materials, vol. 23, no. 6, pp. 383-388, 1999.

[3] A. L. Bazzan, D. Cagara, and B. Scheuermann, "An evolutionary approach to traffic assignment," in Computational Intelligence in Vehicles and Transportation Systems (CIVTS), 2014 IEEE Symposium on. IEEE, 2014, pp. 43-50.

[4] Y.-C. Chiu, "Traffic scheduling simulation and assignment for area-wide evacuation," in Intelligent Transportation Systems, 2004. Proceedings. The 7th International IEEE Conference on. IEEE, 2004, pp. $537-542$.

[5] Q. Lu and S. Shekhar, "Capacity constrained routing for evacuation planning," in Intelligent Transportation Systems Safety and Security Conference (Miami, Florida), USDOT, 2004.

[6] Y.-C. Chiu, J. Bottom, M. Mahut, A. Paz, R. Balakrishna, T. Waller, and J. Hicks, "Dynamic traffic assignment: A primer," Transportation Research E-Circular, no. E-C153, 2011.

[7] S. Peeta and A. K. Ziliaskopoulos, "Foundations of dynamic traffic assignment: The past, the present and the future," Networks and Spatial Economics, vol. 1, no. 3, pp. 233-265, 2001.

[8] M. Saadatseresht, A. Mansourian, and M. Taleai, "Evacuation planning using multiobjective evolutionary optimization approach," European Journal of Operational Research, vol. 198, no. 1, pp. 305-314, 2009.

[9] F. Yuan and L. D. Han, "A multi-objective optimization approach for evacuation planning," Procedia Engineering, vol. 3, pp. 217-227, 2010.

[10] A. Stepanov and J. M. Smith, "Multi-objective evacuation routing in transportation networks," European Journal of Operational Research, vol. 198, no. 2, pp. 435-446, 2009.

[11] H. Dezani, R. D. Bassi, N. Marranghello, L. Gomes, F. Damiani, and I. N. Da Silva, "Optimizing urban traffic flow using genetic algorithm with petri net analysis as fitness function," Neurocomputing, vol. 124, pp. 162-167, 2014.

[12] A. E. Eiben and J. E. Smith, Introduction to Evolutionary Computing, 2nd ed. Spinger, 2015.

[13] A. J. Horowitz, Delay/Volume Relations for Travel Forecasting Based Upon the 1985 Highway Capacity Manual. Federal Highway Administration, U.S. Department of Transportation, 1991.

[14] J. Ortúzar and L. G. Willumsen, Modelling Transport, 3rd ed. John Wiley \& Sons, 2001.

[15] OpenStreetMap contributors, "OpenStreetMap," https://www.openstreetmap.org, 2017. 